\title{
BMJ Open Metformin for the treatment of breast cancer: protocol for a scoping review of randomised clinical trials
}

\author{
Carolina Fumico Massuda Araujo (D) , ${ }^{1}$ Lélia Cápua Nunes (D) ,' \\ Cristiane Murta-Nascimento (D) ,' Cristiano de Pádua Souza (D) ,2 \\ Arinilda Campos Bragagnoli (D) ,2 Fernanda Bono Fukushima (D) , 3 \\ Edison Iglesias de Oliveira Vidal (iD ${ }^{4}$
}

To cite: Araujo CFM, Nunes LC, Murta-Nascimento $\mathrm{C}$, et al. Metformin for the treatment of breast cancer: protocol for a scoping review of randomised clinical trials. BMJ Open 2021;11:e044283. doi:10.1136/ bmjopen-2020-044283

- Prepublication history and additional supplemental material for this paper are available online. To view these files, please visit the journal online. (http://dx.doi.org/10.1136/ bmjopen-2020-044283)

Received 31 August 2020 Accepted 27 July 2021

\section{Check for updates}

(C) Author(s) (or their employer(s)) 2021. Re-use permitted under CC BY-NC. No commercial re-use. See rights and permissions. Published by BMJ.

${ }^{1}$ Public Health Department, São Paulo State University (UNESP), Medical School, Botucatu, São Paulo, Brazil

${ }^{2}$ Medial Oncology Department, Barretos Cancer Hospital, Barretos, São Paulo, Brazil ${ }^{3}$ Anaesthesiology Department, São Paulo State University (UNESP), Medical School, Botucatu, São Paulo, Brazil ${ }^{4}$ Internal Medicine Department, São Paulo State University (UNESP), Medical School, Botucatu, São Paulo, Brazil

Correspondence to Carolina Fumico Massuda Araujo;

carolinafumico@gmail.com

\section{ABSTRACT}

Introduction Innovation through the repurposing of generic drugs encloses several advantages when compared with the process of developing new drugs from scratch. Metformin is an established and inexpensive antidiabetic drug for which anticancer properties have been hypothesised. A systematic review of observational studies found promising results for metformin related to breast cancer in women with diabetes. Although the number of randomised clinical trials of metformin for the treatment of breast cancer increased over the last decades, the overall landscape of those studies in this heterogeneous field remains unclear. Hence, we designed the present scoping review protocol to map the literature on randomised clinical trials of metformin in the treatment of breast cancer to determine the value and scope of future systematic reviews on this subject and identify research gaps.

Methods We will search MEDLINE (via PubMed), EMBASE, CENTRAL, LILACS, Web of Science and sources of grey literature. We will include any randomised clinical trial of metformin for the treatment of breast cancer in adult women, and will not impose restrictions regarding context, language or publication date. Two independent reviewers will screen and select studies, and chart the data. We will structure the presentation of our results based on the molecular types of breast cancer, their stages and treatment modalities.

Ethics and dissemination As a literature review, this study is exempt from ethics approval. Findings will be disseminated through presentations in conferences and a peer-reviewed publication.

Open Science Framework registration osf.io/yquba.

\section{INTRODUCTION}

The repurposing of medications is the process of development of new indications for drugs that were initially developed for other purposes. Innovation through the repurposing of generic drugs, in particular, encloses several advantages when compared with the process of developing new drugs from scratch. For example, besides their relatively low cost, they already have
Strengths and limitations of this study

- This protocol gives transparency to the methods and processes that will be used, allows peer review and reduces the possibility of bias and duplication.

- This scoping review will map the literature on randomised clinical trials on the use of metformin for the treatment of breast cancer, determining the scope and value of future systematic reviews on this subject and identifying research gaps.

- We will not impose any restrictions regarding language or publication date for the selection of studies.

- No formal assessment of risk of bias of included studies, or the overall certainty of evidence across studies will be performed.

- We will not perform meta-analyses as a form of quantitative synthesis.

well-established profiles of safety, pharmacodynamics and pharmacokinetics. Indeed, several publications have highlighted the strategic importance of funding research related to the repurposing of generic medications as a unique opportunity to improve clinical outcomes and decrease costs. ${ }^{1}$ The repurposing of any given medication depends on the quality of the evidence lending support to the new indication and systematic reviews play an essential role in this regard.

Metformin is an established antidiabetic drug for which a whole set of new indications have been studied. ${ }^{2}$ It is one of the most commonly prescribed oral antidiabetic drugs in the world, ${ }^{3}$ and in recent years, several potential beneficial effects arising from the use of metformin and unrelated to diabetes treatment have received increasing attention from the scientific community. One of these potential effects includes a role in modulating different types of cancer. ${ }^{2}$

Importantly, a systematic review of 11 observational studies analysing the association 
between the use of metformin by diabetic women and the prognosis of patients with breast cancer found a very relevant protective association in terms of survival for women using that drug (HR: $0.53,95 \%$ CI: 0.39 to 0.71$){ }^{4}$ However, an important criticism of the meta-analyses of observational studies is that they are also subject to bias due to residual confounding within the original studies included in them, and to date, metformin does not have an established role in the treatment of women with breast cancer.

The last few years have witnessed the appearance of several clinical trials evaluating the use of metformin in the treatment of breast cancer. However, the current understanding of breast cancer is that it is not a single disease but rather a heterogeneous group of diseases with different molecular mechanisms. ${ }^{5}$ Moreover, different stages of breast cancer are associated with different treatment modalities (ie, neoadjuvant, adjuvant and palliative therapies), which are associated with a variety of specific outcomes (eg, pathologic complete response, which is specific to studies of neoadjuvant treatment for early or locally advanced disease). Importantly, a recent systematic review attempted to assess the evidence from randomised clinical trials on the effectiveness of metformin in the treatment of breast cancer. ${ }^{6}$ However, that review suffered from various methodological limitations (eg, was not able to identify a few of the eligible studies) was restricted to a limited range of outcomes and did not take into account the different molecular types and stages of breast cancer or the treatment modalities examined in the original studies, which are central to the appropriate interpretation of the effectiveness not only of metformin but of any treatment of breast cancer. Given the many sources of clinical heterogeneity underlying this field, and the lack of clarity regarding the number of randomised clinical trials of metformin in the treatment of breast cancer, as well as on the molecular types, stages of breast cancer and treatment modalities examined in those trials, we designed the present scoping review protocol to map the literature on randomised clinical trials of metformin in the treatment of breast cancer to determine the value and scope of future systematic reviews on this subject and identify research gaps.

\section{METHODS}

\section{Study registration}

The present scoping review protocol was developed taking into consideration the guidance of the Joanna Briggs Institute for conducting systematic scoping reviews ${ }^{7}$ and was registered with Open Science Framework (osf.io/ yquba).

\section{Review questions}

We will seek to answer the following research questions:

1 . What is the extent of the randomised clinical trials literature on the use of metformin in the treatment of breast cancer?
2. What molecular types and stages of breast cancer were examined in those studies?

3. What treatment modalities, regimens and comparators were used in those studies?

4. What outcomes were evaluated in those studies and what were their main findings?

\section{Eligibility criteria}

We used the following 'population, concept and context' framework to delineate our eligibility criteria.

\section{Population}

Our population of interest was adult (aged 18 years or older) women with any type of breast cancer.

\section{Concept}

We will include randomised clinical trials that have implemented any intervention for the treatment of breast cancer that included the use of metformin either alone or in combination with other pharmacological, surgical or radiation treatments. We will include studies that used placebo, standard treatment or other treatments that do not involve the use of metformin as comparator(s)/control. For studies where metformin was used as an intervention in combination with other treatments, comparators should include the same treatments without metformin. We will accept trials in which the unit of randomisation were individuals or clusters of individuals. We will not impose any restrictions regarding the types of outcomes reported.

\section{Context}

We will not impose any restrictions regarding context, language or publication date for the selection of studies.

\section{Searching}

We will search the following electronic bibliographic databases for relevant studies: MEDLINE through PubMed, EMBASE, LILACS, Web of Science and CENTRAL. Our full search strategy is available in the Supplementary Online Appendix.

Besides searching the databases listed above, we will search for grey literature in the following databases: System for Information on Grey Literature in Europe (Open Grey) and National Library of Medicine Bookshelf. Finally, we will also search ClinicalTrials.gov and the WHO International Clinical Trials Registry Platform (ICTRP) for eligible studies.

Two independent researchers will examine the lists of references identified through electronic search. We will also hand-search reference lists of relevant publications, including review articles on the use of repurposed medications for the treatment of breast cancer and of original studies considered eligible for the review. We also intend to contact researchers to request relevant unpublished data whenever applicable.

\section{Selection of studies}

For all studies identified, two authors will independently screen and review the titles and abstracts. The same 
reviewers will examine full versions of potentially relevant studies independently. Where applicable, we will contact the authors of selected studies to ask for additional data. If there are disagreements regarding the inclusion of a study, these will be resolved through discussion with a third reviewer whenever needed. We will use Rayyan software $^{9}$ to manage the screening of references for our review.

\section{Charting the data}

A standardised, prepiloted form will be used to extract data from the included studies. Extracted information will include the following data: authors; title; complete reference; time period when the study was conducted; country where the study took place; type (histological and molecular), subtypes and staging of breast cancer studied; inclusion/exclusion criteria; sample size; characteristics of the population (eg, presence of diabetes, obesity/overweight and metabolic syndrome); study design; details of the interventions, including treatment modality (ie, neoadjuvant, adjuvant or palliative), dose, frequency and duration of metformin treatment, the line of cancer treatment (eg, first-line treatment) and cointerventions (eg, types of chemotherapy); details of comparators and duration of follow-up; outcome measures with the definitions used by the authors; results; conclusions reported by the study authors; research limitations and funding sources. In addition, there will be a field for the registration of other information deemed relevant by the reviewers and a field for possible information to ask the authors. The data charting form will be amended as needed according to the progress of the data charting process. Disagreements about extracted data will be solved by consensus, and an independent reviewer will be consulted if disagreements persist. Data extracted from each study will be stored in word processing documents throughout the duration of the study and organised in digital spreadsheets as needed.

\section{Collating, summarising and reporting results}

We will structure the presentation of our results around the different molecular types of breast cancer, their stages (ie, metastatic, locally advanced and early disease) and treatment modality. We will present our findings in narrative and tabular form guided by the review questions and objective. Besides, this study will be reported as per an adapted version of the PRISMA-ScR statement (Preferred Reporting Items for Systematic reviews and Meta-Analyses extension for Scoping Reviews). ${ }^{10}$ Our adapted PRISMA-ScR checklist comprising the methods of our review is available in a supplementary file.

\section{Patient and public involvement}

Patients and/or the public were not involved in the design, conduct, reporting or dissemination plans of this research.

\section{AMENDMENTS}

Any amendments to this original protocol will be documented in the methods section of the publication of our results.

\section{ETHICS AND DISSEMINATION}

Because of its nature as a literature review, this study is exempt from ethics approval. The results of the review will be disseminated through presentations in conferences and a peer-reviewed publication.

\section{DISCUSSION}

Breast cancer is the leading cause of cancer death in women and the third highest incident cancer globally, claiming around 180 thousand lives and resulting in 17.7 million disability-adjusted life years globally in $2017 .{ }^{11}$ Drug repurposing is an opportunity to minimise risks, costs and time in the development of new pharmacologic treatments while preserving the benefits for healthcare. Although the number of randomised clinical trials of metformin for the treatment of breast cancer increased over the last decades, the overall landscape of those studies and the value and scope of systematic reviews to be conducted based on them remain unclear. In this protocol, we outline the details of the aims and methods of a scoping review of randomised controlled trials of metformin for the treatment of breast cancer. We hope that, once completed, this scoping review may contribute to the field of drug repurposing related to cancer by mapping the available evidence from randomised clinical trials and identifying research gaps related to the use of metformin in breast cancer.

Acknowledgements The authors wish to express their gratitude to the Office for Research Support at Botucatu Medical School, São Paulo State University (UNESP).

Contributors CFA and EIOV conceived the study, designed the protocol and drafted the manuscript. LCN, CM-N, CdPS, ACB and FF critically revised the protocol and the manuscript. All authors approved the final version of the manuscript.

Funding This work was supported by CAPES (Coordination for the Improvement of Higher Education Personnel-Ministry of Education, Brazil), Process number: 8882.432879/2019-01.

Disclaimer The funder did not play any role in the design of the present protocol or in the decision to publish it.

Competing interests None declared.

Patient consent for publication Not required.

Provenance and peer review Not commissioned; externally peer reviewed.

Supplemental material This content has been supplied by the author(s). It has not been vetted by BMJ Publishing Group Limited (BMJ) and may not have been peer-reviewed. Any opinions or recommendations discussed are solely those of the author(s) and are not endorsed by BMJ. BMJ disclaims all liability and responsibility arising from any reliance placed on the content. Where the content includes any translated material, BMJ does not warrant the accuracy and reliability of the translations (including but not limited to local regulations, clinical guidelines, terminology, drug names and drug dosages), and is not responsible for any error and/or omissions arising from translation and adaptation or otherwise.

Open access This is an open access article distributed in accordance with the Creative Commons Attribution Non Commercial (CC BY-NC 4.0) license, which permits others to distribute, remix, adapt, build upon this work non-commercially, 
and license their derivative works on different terms, provided the original work is properly cited, appropriate credit is given, any changes made indicated, and the use is non-commercial. See: http://creativecommons.org/licenses/by-nc/4.0/.

\section{ORCID iDs}

Carolina Fumico Massuda Araujo http://orcid.org/0000-0003-4713-5749

Lélia Cápua Nunes http://orcid.org/0000-0002-2651-7572

Cristiane Murta-Nascimento http://orcid.org/0000-0001-8686-1079

Cristiano de Pádua Souza http://orcid.org/0000-0002-6412-8041

Arinilda Campos Bragagnoli http://orcid.org/0000-0002-5165-0797

Fernanda Bono Fukushima http://orcid.org/0000-0001-8055-5425

Edison Iglesias de Oliveira Vidal http://orcid.org/0000-0002-1573-4678

\section{REFERENCES}

1 Sachs RE, Ginsburg PB, Goldman DP. Encouraging new uses for old drugs. JAMA 2017;318:2421-2.

2 Abdelgadir E, Ali R, Rashid F, et al. Effect of metformin on different Non-Diabetes related conditions, a special focus on malignant conditions: review of literature. J Clin Med Res 2017:9:388-95.

3 Santomauro Júnior AC, Ugolini MR, Santomauro AT, et al. [Metformin and AMPK: an old drug and a new enzyme in the context of metabolic syndrome]. Arq Bras Endocrinol Metabol 2008;52:120-5.
$4 \mathrm{Xu} \mathrm{H}$, Chen $\mathrm{K}$, Jia X, et al. Metformin use is associated with better survival of breast cancer patients with diabetes: a meta-analysis. Oncologist 2015;20:1236-44.

5 Testa U, Castelli G, Pelosi E. Breast cancer: a molecularly heterogenous disease needing subtype-specific treatments. Med Sci 2020;8. doi:10.3390/medsci8010018. [Epub ahead of print: 23 Mar 2020].

6 Zhang Z-J, Yuan J, Bi Y, et al. The effect of metformin on biomarkers and survivals for breast cancer- a systematic review and meta-analysis of randomized clinical trials. Pharmacol Res 2019;141:551-5.

7 Peters MDJ, Godfrey CM, Khalil H, et al. Guidance for conducting systematic scoping reviews. Int J Evid Based Healthc 2015;13:141-6.

8 Araujo CFM, Nunes LC, Murta-Nascimento C. Metformin for the treatment of breast cancer: protocol for a scoping review of randomised clinical trials, 2021. Available: https://osf.io/g3jaz [Accessed 12 Mar 2021].

9 Ouzzani M, Hammady H, Fedorowicz Z, et al. Rayyan-a web and mobile APP for systematic reviews. Syst Rev 2016;5:210.

10 Tricco AC, Lillie E, Zarin W, et al. PRISMA extension for scoping reviews (PRISMA-ScR): checklist and explanation. Ann Intern Med 2018;169:467-73.

11 Li N, Deng Y, Zhou L, et al. Global burden of breast cancer and attributable risk factors in 195 countries and territories, from 1990 to 2017: results from the global burden of disease study 2017. $J$ Hematol Oncol 2019;12:140. 